\title{
Perfil do biodiesel B100 comercializado na região de Londrina: aplicação de redes neurais do tipo mapa auto-organizável
}

\section{Profile of the biodiesel B100 commercialized in the region of Londrina: application of artificial neural networks of the type self organizing maps}

\author{
Vilson Machado de Campos Filho ${ }^{1}$; Kelly Roberta Spacino²; Érica Signori Romagnoli; \\ Lívia Ramazzoti Chanan Silva ${ }^{4}$; Dionisio Borsato ${ }^{5^{*}}$
}

\section{Resumo}

As 97 amostras foram agrupadas de acordo com o ano de análise. Para cada ano foram atribuídas letras de A à D, compreendidas entre 2010 e 2013; A (33), B (25), C (24) e D (15). Dos parâmetros de conformidade previamente analisados, encontram-se os preconizados pela Agência Nacional de Petróleo, Gás Natural e Biocombustíveis (ANP), por meio da resolução ANP 07/2008. Dentre os parâmetros foram analisados a massa específica, ponto de fulgor, índice de peróxido e índice de acidez. Os valores observados foram apresentados a Rede Neural Artificial (RNA) do tipo Mapa Auto Organizável (MAO) a fim de obter uma classificação de acordo com as propriedades físico - químicas de cada amostra e seu ano de produção. A RNA foi treinada em dias diferentes e as amostras divididas aleatoriamente em dois grupos, treinamento e teste. Foi constatado que para esta situação, a rede MAO diferenciou as amostras em relação ao ano e aos parâmetros de conformidade, permitindo a constatação de que dentre os parâmetros de conformidade analisados, os de maior significância, portanto, bons para a distinção e classificação dessas amostras, foram a massa específica e o ponto de fulgor.

Palavras-chave: Redes Neurais Artificiais. Mapas Auto Organizáveis. Parâmetros de Conformidade. Biocombustível. Biodiesel.

\begin{abstract}
The 97 samples were grouped according to the year of analysis. For each year, letters from A to D were attributed, between 2010 and 2013; A (33) B (25) C (24) and D (15). The parameters of compliance previously analyzed are those established by the National Agency of Petroleum, Natural Gas and Biofuels (ANP), through resolution ANP 07/2008. The parameters analyzed were density, flash point, peroxide and acid value. The observed values were presented to Artificial Neural Network (ANN) Self Organizing MAP (SOM) in order to classify, by physical-chemical properties, each sample from year of production. The ANN was trained on different days and randomly divided samples into two groups, training and test set. It was found that SOM network differentiated samples by the year and the compliance parameters, allowing to identify that the density and the flash point were the most significant compliance parameters, so good for the distinction and classification of these samples.
\end{abstract}

Keywords: Artificial Neural Networks. Self Organizing Maps. Compliance parameters. Biofuel. Biodiesel.

\footnotetext{
${ }^{1}$ Mestrando em Bioenergia, Universidade Estadual de Londrina; vyllson@hotmail.com

${ }^{2}$ Mestranda em Bioenergia, Universidade Estadual de Londrina;

${ }^{3}$ Acadêmica de Graduação em Química, Universidade Estadual de Londrina;

${ }^{4}$ Acadêmica de Graduação em Química, Universidade Estadual de Londrina;

${ }^{5}$ Departamento de Química, Universidade Estadual de Londrina; *dborsato@uel.br
} 


\section{Introdução}

No Brasil, a proposta da substituição dos combustíveis de origem fóssil, pelos obtidos através da biomassa, ganhou força com crise petrolífera da década de 1970 e o seu marco deuse com a criação do Programa Nacional do Álcool (PRÓÁLCOOL), que tornava real a substituição da gasolina pelo álcool combustível (TEIXEIRA; TAOUIL, 2010).

Visando estreitar ainda mais a dependência dos combustíveis derivados do petróleo e como alternativa consolidada para a substituição do diesel de origem fóssil, a utilização de óleos de origem vegetal é motivada pela produção do biodiesel, cujo, testes realizados apresentam resultados favoráveis e viáveis, isso também se considerarmos a capacidade produtiva nacional (TEIXEIRA; TAOUIL, 2010), (OSAKI; BATALHA, 2011).

O biodiesel é uma denominação genérica para combustíveis produzidos a partir de fontes renováveis, como óleos vegetais e gorduras animais, para serem utilizados em motores de ignição por compressão, também conhecidos como motores diesel (BORSATO et al., 2010), (CUNHA JR et al., 2013).

Outro eixo norteador para o emprego da substituição dos combustíveis de origem fóssil é a questão ambiental, que prevê a redução e melhorias na gestão de resíduos, bem como da redução de gases nocivos ao meio ambiente e a qualidade de vida, em particular, à atmosfera (ORIVES et. al., 2014).

Dentre as exigências preconizadas pela Agência Nacional de Petróleo, Gás Natural e Biocombustíveis (ANP), quanto aos parâmetros de conformidade do biodiesel, encontram-se os que são provenientes da normatização do diesel mineral e os que foram originados dos óleos de origem vegetal, comumente utilizados na indústria óleoquímica (LÔBO; FERREIRA, 2009), (BRASIL, 2012).

Diante das exigências preconizadas, fazse necessária a otimização do processo em termos qualitativos e quantitativos, conferindo agilidade e segurança no controle de qualidade do biodiesel. Nos processos industriais, os modelos computacionais "inteligentes" tornaram-se muito importantes e têm sido usados em todas as etapas desde a produção até a segmentação de mercado (CORNEY, 2002).

Uma ferramenta que tem se mostrado muito adequada nos tratamentos de dados que possuem comportamento não linear são as redes neurais artificiais (RNA). Existem vários tipos de redes neurais tais como, perceptron de uma ou múltiplas camadas, redes de base radial, mapas autoorganizáveis, dentre outros (BORSATO et. al., 2011) (CREMASCO, 2014).

A utilização das RNA permite obter não somente ganhos significativos de desempenho, mas também possibilita o desenvolvimento de aplicações inovadoras capazes de expandir nossos sentidos e habilidades intelectuais e, com isso, permite avaliar a influência de cada atributo auxiliando os especialistas em suas tomadas de decisões (HAYKIN, 2001).

Os mapas auto-organizáveis de Kohonen são um tipo de rede neural artificial baseada em aprendizado competitivo e não supervisionado, sendo capaz de mapear um conjunto de dados de um espaço de entrada contido em um conjunto finito de neurônios organizados em um arranjo normalmente unidimensional ou bidimensional. Por fornecer uma aproximação discreta das chamadas superfícies principais, eles são adequados para a tarefa de seleção de características. (HAYKIN, 2001).

O principal objetivo dos Mapas Autoorganizáveis (MAO) de Kohonen é agrupar os dados de entrada que são semelhantes entre si formando classes ou agrupamentos denominados clusters (KOHONEN, 1997; FONSECA et al., 2006). Para um uso mais racional do MAO é importante o emprego de ferramentas estatísticas para avaliar tanto a preservação da topologia inerente aos dados, como a variabilidade do erro de quantização e estabilidade das relações de vizinhança (BODT; COTTRELL; VERLEYSEN 2002).

Este trabalho teve como objetivo aplicar e adaptar a metodologia de redes neurais artificiais (RNA) para classificar amostras de biodiesel B100, tendo como parâmetros a massa específica, ponto de fulgor, índice de peróxido e índice de acidez. 


\section{Materiais e Métodos}

\section{Amostragem}

Foram analisadas 97 amostras de biodiesel B100 comercializadas na região de Londrina-PR, durante o período compreendido entre janeiro de 2010 a julho de 2013 sendo de uma a três amostras por mês coletadas em dias diferentes. Essas amostras foram submetidas aos ensaios de massa específica a $20^{\circ} \mathrm{C}$, ponto de fulgor, índice de acidez e índice de peróxido. Os valores médios tabulados, em triplicata, foram apresentados à rede neural do tipo MAO para classificação e estabelecimento do perfil das amostras comercializadas na região de Londrina-PR. As amostras foram identificadas com as letras, onde cada letra corresponde ao ano de analise; A (33) de 2010, B (25) de 2011, C (24) de 2012 e D (15) de 2013 e o número entre parênteses indica a quantidade de amostras analisadas para cada ano.

Massa especifica.

A determinação da massa específica foi realizada de acordo com a norma NBR 7148.

\section{Ponto de Fulgor}

A determinação do ponto de fulgor foi realizada com o Fulgor Full Control, modelo PD 500, Petrodidática, de acordo com a norma NBR 14598.

\section{Índice de Acidez.}

A determinação do índice de acidez foi realizada através do método de titulação, de acordo com a norma NBR 14448.

\section{Índice de Peróxido}

A determinação do índice de peróxido foi realizada através de titulação, com tiossulfato de sódio pelo método A. O. A. C. Cd 8-53 (A. O. C. S., 1998).

\section{Redes Neurais Artificiais.}

Neste trabalho foi implementado o algoritmo MAO bidimensional, no qual o mapa autoorganizável transforma um padrão de sinal incidente de dimensão arbitrária em um mapa discreto bidimensional realizando esta transformação de uma maneira topologicamente ordenada.

Para testar as duas principais fontes de variabilidade das redes foi utilizado primeiramente o gerador de número aleatório igual a 250 e depois 500 para cada MAO com ciclos de 250, 500, 750 e 1000. Com o mesmo gerador de número aleatórios, cada rede foi treinada 10 vezes para verificar se as propriedades topológicas estavam sendo preservadas (BODT; COTTRELL; VERLEYSEN, 2002).

Foi utilizado o módulo de redes neurais artificiais do software Statistica 9 (STATISTICA, 2009). A ordem de entrada dos parâmetros na rede foram os valores da massa específica, ponto de fulgor, índice de acidez e índice de peróxido. Foi utilizada uma rede do tipo MAO.

\section{Análise estatística}

O teste de Tukey e Levene's e os desvios padrão foram estimados por meio do software Statistica v.9.

\section{Processamento}

Todos os resultados dos experimentos foram processados em um computador Intel Pentium Core 2 Duo de $1,8 \mathrm{GHz}$ e 2 GB de memória RAM.

\section{Resultados e Discussão}

As Figuras 1a, 1b, 1c e 1d mostram os valores obtidos para a massa específica $\left(\mathrm{kg} \mathrm{m}^{-3}\right)$, ponto de fulgor $\left({ }^{\circ} \mathrm{C}\right)$, índice de acidez $\left(\mathrm{mg}_{\mathrm{Kон}} \mathrm{g}^{-1}\right)$ e índice de peróxido $\left(\mathrm{m}_{\mathrm{eq}} \mathrm{kg}\right)$, respectivamente das 97 amostras analisadas. As amostras foram separadas 
por ano de amostragem indicadas pelas linhas verticais e as linhas horizontais indicam os limites dos parâmetros de conformidade de acordo com a legislação Brasileira vigente na época.

A legislação vigente no Brasil, especificada na Resolução da ANP $n^{\circ} 14$ de 11.05.2012 DOU 18.05.2012, estabelece os parâmetros de conformidade para o biodiesel B100, sendo para a massa específica de 850 a $900 \mathrm{~kg} \mathrm{~m}^{-3}$, ponto de fulgor de $100^{\circ} \mathrm{C}$ mínimo, índice de acidez máximo de $0,5 \mathrm{mg}_{\mathrm{KOH}} \mathrm{g}^{-1}$. E para o índice de peróxido, de acordo com BORSATO et al. (2010), recomenda-se utilizar um biodiesel B100 quando o seu respectivo valor encontra-se abaixo de $15 \mathrm{~m}_{\mathrm{eq}} \mathrm{kg}^{-1}$.

Figura 1 - Massa específica $\left(\mathrm{kg} \mathrm{m}^{-3}\right)(\mathrm{A})$, ponto de fulgor $\left({ }^{\circ} \mathrm{C}\right)(\mathrm{B})$, índice de acidez $\left(\mathrm{mg}_{\mathrm{KOH}} \mathrm{g}^{-1}\right)(\mathrm{C})$ e índice de peróxido $\left(\mathrm{m}_{\mathrm{eq}} \mathrm{kg}^{-1}\right)$ (D) das amostras analisadas.
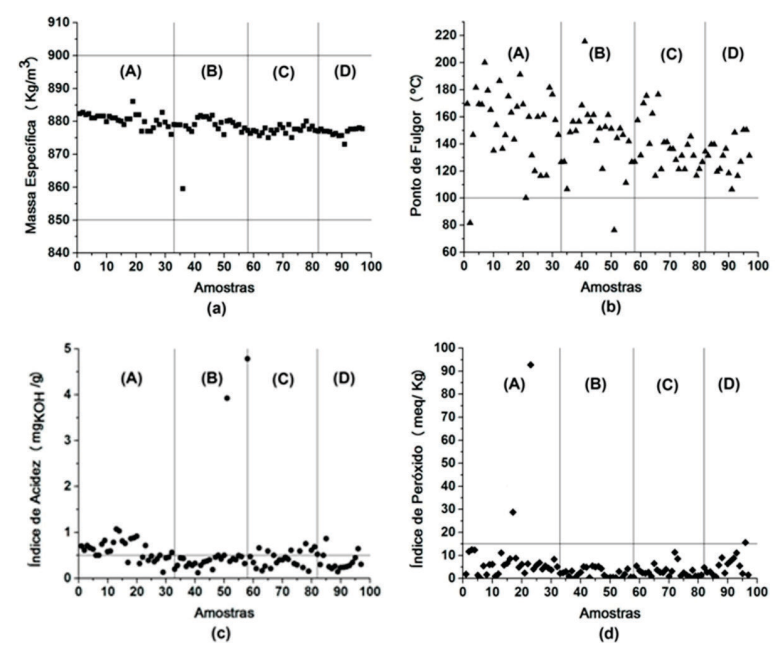

Fonte: Autor.

A Figura 1a mostra que todas as amostras analisadas estavam de acordo com a legislação vigente para o parâmetro de massa específica isto é, entre 850 a $900 \mathrm{~kg} / \mathrm{m}^{3}$. A Figura $1 \mathrm{~b}$ representa os valores obtidos para o ponto de fulgor e abaixo da linha horizontal que indica o valor mínimo estabelecido $\left(100^{\circ} \mathrm{C}\right)$, apenas três amostras apresentaram-se fora dos parâmetros de conformidade, duas em 2010 e uma em 2011. Na Figura 1c a linha horizontal indica o limite máximo para o índice de acidez, estabelecidas em $0,5 \mathrm{mg}$ $\mathrm{g}^{-1}$, na qual é possível verificar que trinta amostras se encontram fora da especificação sendo 19 delas em 2010. A Figura 1d mostra os resultados da análise de índice de peróxido sendo que apenas três amostras (2 em A e 1 em D) apresentaram-se fora dos parâmetros de conformidade recomendados, estabelecidos pelo limite máximo de $15 \mathrm{~m}_{\mathrm{eq}} \mathrm{kg}^{-1}$.

A elevada acidez é causada pelos ácidos graxos livres que quando provindos da matéria-prima podem dificultar ou até invibializar a formação dos ésteres quando se trata de catálise homogêna em meio alcalino (transesterificação). Quanto ao índice de peróxidos, vale ressaltar que o parâmetro refere-se a compostos intermediários da degradação lipídica, podendo haver lipídios bastante degradados com baixos teores de peróxidos, por isso esse parâmetro não deve ser avaliado isoladamente, especialmente quando há uma considerável quantidade de amostras com acidez elevada.

Como as especificações estabelecidas pela ANP não foram utilizadas para fim de treinamento da rede, isto é, não foram informadas para a rede utilizada, o fato delas estarem fora de especificação não vai influenciar na decisão tomada pela RNA utilizada.

O programa utilizado apresenta a opção de resolver as RNA utilizando o módulo automático, isto é, ele escolhe o numero de épocas, taxa de aprendizagem, relação de vizinhança mais adequadas para cada rede. A rede MAO, do módulo automático de redes neurais do STATISTICA 9 foi alimentada com os parâmetros de massa específica, ponto de fulgor, índice de acidez e peróxido, totalizando 97 amostras.

As amostras de biodiesel B100 dos diferentes anos foram divididas, aleatoriamente, em duas partes sendo que a primeira representando o conjunto de treinamento da rede foi formada com $70 \%$ das amostras. O segundo grupo chamado de teste foi composto com $30 \%$ das amostras, sendo que este conjunto teve como objetivo produzir saídas adequadas para entradas que não estavam 
presentes durante o treinamento, e com isso validar a sua capacidade de aprendizagem. O parâmetro de vizinhança começou com um valor inicial igual a três diminuindo essencialmente para zero e o parâmetro da taxa de aprendizagem iniciou com valor inicial de $10 \%$ decaindo para $2 \%$ (HAYKIN, 2001).

Foi possível observar (Figura 2) que a topologia 4 x 7, com a aplicação de números aleatórios de geração de dados iguais ou maiores que 500 e ciclos de treinamento igual a 750, foi a mais adequada para os dados analisados, porque a variação de erros obtida ficou entre $0,0202 \%$ e $0,0223 \%$ para treinamento e $0,0811 \%$ e $0,0982 \%$ para teste.

A Figura 2 indica que de 630 a 750 ciclos, durante a fase de ordenação, são suficientes para realizar o treinamento da rede com a topologia 4 x 7. Entretanto, foi escolhido 750 ciclos devido ao fato de que a partir deste valor os mapas não sofreram mais modificações quanto à disposição e à vizinhança das amostras.

Figura 2 - Gráfico do erro obtido para o treinamento e teste em relação ao número de ciclos de treinamento da rede com topologia $4 \times 7$.

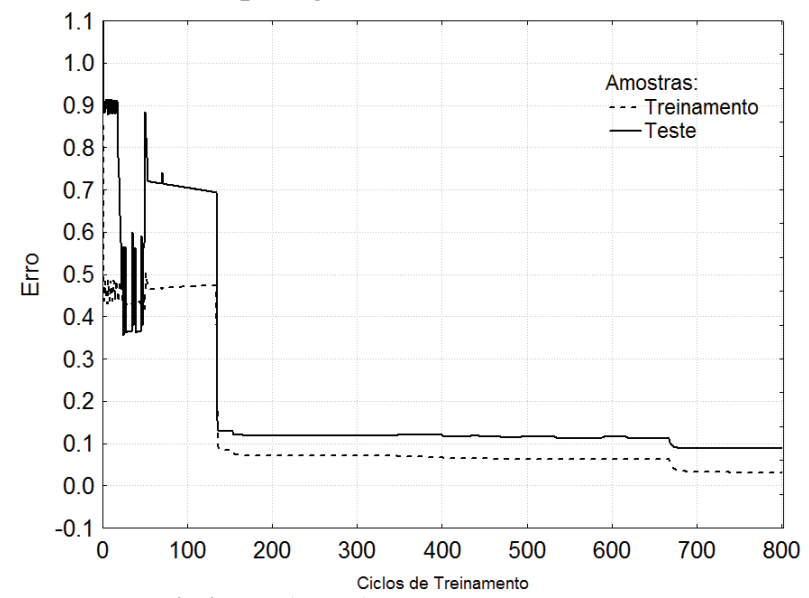

Fonte: Statistica 9 (2009).

A Tabela 1 mostra o valor máximo e mínimo, valor médio e desvio padrão de cada parâmetro utilizado para treinamento e teste de uma das redes utilizadas.
$\mathrm{Na}$ classificação e identificação do ano, foram utilizados 750 ciclos de treinamento e 500 como número aleatório de geração de dados. Este valor inteiro produz as amostras aleatórias, que para criar uma amostragem diferente ele deve ser alterado. Habitualmente, o grau em que duas ou mais variáveis independentes estão relacionados com a variável dependente é expressa no coeficiente de correlação $\left(\mathrm{R}^{2}\right)$. Para o caso em estudo o valor do $R^{2}$ foi igual a 0,21 . Portanto, presume-se que a relação entre as variáveis é não linear e com isso as redes de Kohonen podem ser aplicadas para o caso, pois, de acordo com Ritter (1995), um mapa autoorganizável é inerentemente não linear.

Tabela 1 - Dados estatísticos de cada parâmetro utilizado para treinamento e teste de uma das redes utilizadas quando se utilizou 500 como número aleatório de geração de dados.

\begin{tabular}{lcccl}
\hline Amostras & $\begin{array}{c}\text { Massa } \\
\text { específica } \\
\left(\mathbf{k g ~ m}^{-3}\right)\end{array}$ & $\begin{array}{c}\text { Ponto de } \\
\text { Fulgor } \\
\left({ }^{\circ} \mathbf{C}\right)\end{array}$ & $\begin{array}{c}\text { Índice de } \\
\text { Acidez } \\
(\mathbf{m g K O H} \\
\left.\mathbf{g}^{-1}\right)\end{array}$ & $\begin{array}{l}\text { Índice de } \\
\text { Peróxido } \\
\left(\mathbf{m}_{\mathrm{eq}} \mathbf{~ k g}^{-1}\right)\end{array}$ \\
\hline $\begin{array}{l}\text { Treinamento } \\
\text { Mínimo }\end{array}$ & 873,00 & 76,30 & 0,12 & 0,26 \\
Máximo & 886,00 & 215,50 & 4,78 & 92,70 \\
Média & 878,59 & 143,07 & 0,56 & 5,29 \\
Desvio & & & & \\
padrão & 2,20 & 23,99 & 0,70 & 11,48 \\
Teste & & & & \\
Mínimo & 859,50 & 81,60 & 0,19 & 0,08 \\
Máximo & 882,70 & 200,00 & 1,03 & 15,62 \\
Média & 878,42 & 146,84 & 0,50 & 5,33 \\
Desvio & & & & \\
padrão & 4,27 & 24,50 & 0,22 & 4,28 \\
Geral & & & & \\
Mínimo & 859,50 & 76,30 & 0,12 & 0,08 \\
Máximo & 886,00 & 215,50 & 4,78 & 92,70 \\
Média & 878,54 & 144,20 & 0,54 & 5,31 \\
Desvio & & & & 9,86 \\
padrão & 2,95 & 24,08 & 0,60 & \\
\hline Fonte: Statica & & & \\
\hline
\end{tabular}

Fonte: Statistica (2009).

Para avaliar a existência ou não de diferença significativa entre as amostras dos diferentes anos, foi gerada a Tabela 2 que nos mostra o valor médio, o número de amostras utilizadas, o desvio padrão e a comparação entre os valores médios obtidos para cada parâmetro analisado, 
utilizando o teste de Tukey. Letras iguais mostram que não há diferença significativa entre as médias obtidas para cada parâmetro das amostras analisadas nos diferentes anos. Além disso, de acordo com o teste de Levene's, como o menor valor de p observado foi de cerca $5 \%$ $\leq \mathrm{p} \geq 11,68 \%$ a hipótese de homogeneidade de variância em nível de 5\% pode ser aceita (STATISTICA, 2009).

Tabela 2 - Teste de Tukey aplicado às médias dos parâmetros utilizados na classificação dos diferentes anos de produção das amostras de biodiesel B100.

\begin{tabular}{ccccc}
\hline Ano & $\begin{array}{c}\text { Massa } \\
\text { Específica } \\
\left(\mathbf{K g ~ m}^{-3}\right)\end{array}$ & $\begin{array}{c}\text { Fulgor } \\
\left({ }^{\circ} \mathbf{C}\right)\end{array}$ & $\begin{array}{c}\text { Índice } \\
\mathbf{d e} \text { acidez } \\
\left(\mathbf{m g}_{\text {KOH }}\right. \\
\left.\mathbf{g}^{-1}\right)\end{array}$ & $\begin{array}{c}\text { Índice de } \\
\text { Peróxido } \\
(\mathbf{m e q} \\
\left.\mathbf{K g}^{-1}\right)\end{array}$ \\
\hline $\mathbf{A}$ & $880,43^{\mathrm{a}}$ & $154,06^{\mathrm{a}}$ & $0,60^{\mathrm{a}}$ & $8,92^{\mathrm{a}}$ \\
$\mathbf{N}=\mathbf{3 3}$ & $(0,36)$ & $(4,71)$ & $(0,04)$ & $(2,78)$ \\
$\mathbf{B}$ & $878,36^{\mathrm{b}}$ & $144,50^{\mathrm{ab}}$ & $0,68^{\mathrm{a}}$ & $2,33^{\mathrm{a}}$ \\
$\mathbf{N}=\mathbf{2 5}$ & $(0,85)$ & $(5,14)$ & $(0,22)$ & $(0,38)$ \\
$\mathbf{C}$ & $877,29^{\mathrm{b}}$ & $138,47^{\mathrm{ab}}$ & $0,42^{\mathrm{a}}$ & $3,27^{\mathrm{a}}$ \\
$\mathbf{N = \mathbf { 2 4 }}$ & $(0,25)$ & $(3,65)$ & $(0,04)$ & $(0,53)$ \\
$\mathbf{D}$ & $876,70^{\mathrm{b}}$ & $131,15^{\mathrm{b}}$ & $0,35^{\mathrm{a}}$ & $5,56^{\mathrm{a}}$ \\
$\mathbf{N = 1 5}$ & $(0,33)$ & $(3,42)$ & $(0,05)$ & $(1,10)$ \\
\hline
\end{tabular}

Fonte: Statistica (2009).

O teste de Tukey aplicado às médias (Tabela 2) mostrou que para a massa específica, somente para as amostras do ano A existe diferença significativamente maior, em nível de 5\%, em relação às demais. Para o ponto de fulgor existe diferença significativa apenas entre os anos A e D. Para o índice de acidez e índice de peróxido não foram observados nenhuma diferença significativa entre os anos considerados.

A Figura 3 mostra os mapas de duas das 10 repetições do treinamento e teste, utilizando a topologia $4 \times 7$, onde cada quadrado representa um neurônio. A cada inicialização do aplicativo, em dias diferentes, observou-se que sempre foi obtido o mesmo mapa e seu respectivo espelho (Figura 3a e 3b) e em todos os casos, as condições de vizinhança para o treinamento e teste foram respeitadas.
Figura 3 - Mapas topológicos de duas repetições para o treinamento e teste da rede utilizada. A, B, C, D representam amostras dos anos 2010, 2011, 2012 e 2013 respectivamente.
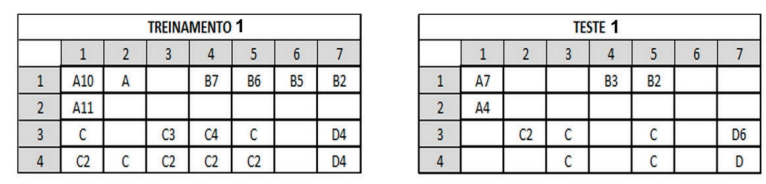

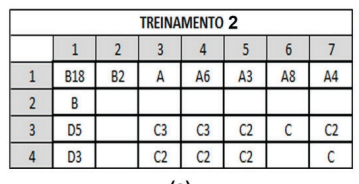

(a)

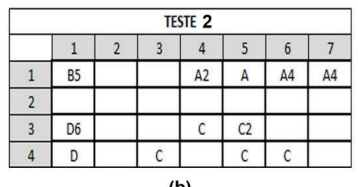

(b)
Fonte: Autor.

Em todas as repetições da topologia utilizada, apesar do algoritmo responsável pela formação do MAO inicializar os pesos sinápticos aleatoriamente sem nenhuma imposição de organização prévia, podemos verificar que os agrupamentos, a cada inicialização, mesmo em posições diferentes, mantiveram a mesma relação de vizinhança mostrando, com isso, a estabilidade da rede utilizada e da preservação das propriedades topológicas (MELSSEN; WEHRENS; BUYDENS, 2006). Isto indica que a rede empregada, na topologia escolhida, consegue discriminar as amostras de acordo com o ano de analise.

Em todos os mapas obtidos foi possível verificar uma separação entre as amostras do ano A e D. A única amostra que permaneceu agrupada, tanto para treinamento quanto para teste, em pelo menos um conjunto de mapas, foi a amostra D. Apesar das amostras B, C e D mudarem de posição nos dois mapas de treinamento apresentados elas mantiveram a mesma relação de vizinhança, assim como, A, B e C.

É possível observar que as amostras $\mathrm{A}$ e C, apresentam uma maior dispersão indicando que os parâmetros de conformidade analisados não tiveram padrões de qualidade semelhantes.

De acordo com a Tabela 2, as amostras dos anos A e D apresentam diferença significativa 
nos parâmetros de massa especifica e ponto de fulgor, similaridade essa comprovada pelos mapas mostrados na Figura 3, na qual as mesmas amostras estão distantes uma da outra.

As amostras A, B, e C não apresentaram diferença significativa nos parâmetros analisados, exceto quanto à massa específica, que faz com que elas mantenham-se uma vizinhança próxima, fato este que também deve explicar a dispersão e o agrupamento de $\mathrm{A}$ em regiões de diferentes mapas.

\section{Conclusões}

Ametodologia empregada no uso do MAO para as 97 amostras de biodiesel B100 analisadas mostrouse de suma relevância para o reconhecimento de padrões significativos à diferenciação e classificação do perfil desse biodiesel comercializado na região de Londrina. Para este caso o módulo da RNA utilizada permitiu a diferenciação das amostras de acordo com seu ano de produção.

\section{Referências}

ASSOCIAÇÃO BRASILEIRA DE NORMAS TÉCNICAS - ABNT. NBR 7148: Petróleo e derivados de petróleo - Determinação da massa específica, densidade relativa $\mathrm{e}^{\circ} \mathrm{API}$ - Método do densímetro. Rio de Janeiro, 2013.

.NBR 14598: Produtos de petróleo - determinação do ponto de fulgor pelo aparelho de vaso fechado PenskyMartens. Rio de Janeiro, 2012.

. NBR 14448: Óleos lubrificantes, produtos de petróleo e biodiesel - determinação do número de acidez pelo método de titulação potenciométrica. Rio de janeiro, 2013.

AGÊNCIA NACIONAL DE PETRÓLEO, GÁS NATURAL E BIOCOMBUSTÍVEIS - ANP. Boletim Mensal do Biodiesel, 2014. Superintendência de Refino, Processamento de Gás Natural e Produção de Biocombustíveis. Brasília, DF, 2014.
BODT, E.; COTTRELL, M.; VERLEYSEN, M. Statistical tools to assess the reliability of self-organizing maps. Neural Networks, v.15, p.967-978, 2002.

BORSATO, D.; DALL'ANTONIA, L. H.; GUEDES, C. L. B.; MAIA, E. C. R.; FREITAS, H. R.; MOREIRA, I.; SPACINO, K. R. Aplicação do delineamento simplexcentroide no estudo da cinética da oxidação de biodiesel B100 em mistura com antioxidantes sintéticos. Quimica Nova, v.33, n.8, p.1726-1731, 2010

BORSATO, D.; PINA, M. V. R.; SPACINO, K. R.; SCHOLZ, M. B. S.; ANDROCIOLI, A. Application of artificial neural betworks in the geographical identification of coffee samples. European Food Research \& Technology, v.233, n.3, p. 533-543, 2011

BRASIL. Ministério de Minas e Energia. Agência Nacional do Petróleo, Gás Natural e Biocombustíveis. Estabelece a especificação para comercialização de biodiesel que poderá ser adicionado ao óleo diesel. Resolução n. ${ }^{0}$ 14, de 11 de maio de 2012. Diário Oficial da União, Brasília, 2012.

GÁS NATURAL E COMBUSTÍVEIS RENOVÁVEIS. Boletim mensal dos combustíveis renováveis. Resolução $\mathrm{n}^{\circ} 80$, de 8 de outubro de 2014, publicado no Diário Oficial da União, Brasília, DF, 2014.

CORNEY D. Food bytes: intelligent systems in food industry. British Food Journal, v.104, n.10, p.787-805, 2002 .

CREMASCO, H. Aplicação de redes neurais artificiais do tipo mapas auto-organizáveis: estudo de casos. 2014. Dissertação (Mestrado em Química) - Universidade Estadual de Londrina, Londrina.

CUNHA JR, A.; FEDDERN, V.; DE PRÁ, M. C.; HIGARASHI, M. M.; ABREU, P. G.; COLDEBELLA, A. Synthesis and characterization of ethylic biodiesel from animal fat wastes. Fuel, v.105, n.3, p.228-234, 2013.

FONSECA, A. M.; BISCAYA, J. L.; AIRES DE SOUZA, J.; LOBO, A. M. Geographical classification of crude oils by Kohonen self-organizing maps. Analytica Chimica Acta, v. 556, n. 2, p. 374-382, 2006.

HAYKIN, S. Redes Neurais: princípios e práticas. 2. ed. Porto Alegre: Bookman, 2001. 
KOHONEN, T. Self organizing maps. $2^{\text {th }}$ ed. Verlag, Heidelberg: Springer, 1997. (Series in Information Sciences, v. 30).

LÔBO, I. P.; FERREIRA, S. L. C. Biodiesel: parâmetros de qualidade e métodos analíticos. Química Nova, v. 32, n. 6, p. 1596-1608, 2009.

MELSSEN, W.; WEHRENS, R.; BUYDENS, L. Supervised Kohonen networks for classification problems. Chemometrics and Intelligent Laboratory Systems, v. 83, n. 2, p. 99-113, 2006.

OFFICIAL METHODS AND RECOMMENDED PRACTICES OF THE AMERICAN OIL CHEMIST'S SOCIETY - AOAC. American Oil Society. $4^{\text {th }}$ ed. Champaign: AOAC, 1997. Method Cd 8-53.

ORIVES, J. R.; GALVAN, D.; COPPO,R. L.; RODRIGUES, C. H. F.; ANGILELLI, K. G.; BORSATO, D. Multiresponse optimisation on biodiesel obtained through a ternary mixture of vegetable oil and animal fat: simplex-centroid mixture design application. Energy Conversion and Management, n. 79, n. 1, p. 398-404, 2014.

OSAKI, M.; BATALHA, M. O. Produção de Biodiesel e Óleo Vegetal no Brasil: Realidade e Desafio. Organizações Rurais \& Agroindustriais, v. 13, n. 2, p. 227-242, 2011.

RITTER, H. Self-organizing feature maps: Kohonen maps. In: ARBIB, M. A. (ed). The handbook of brain theory and neural network. Cambridge, MA: MIT Press, 1995. p. 846-851.

STATISTICA. Graphics software. Tulsa: Statistica For Windows, 2009. v. 9.

TEIXEIRA, M. C.; TAOUIL, D. S. G. Biodiesel: uma energia alternativa e verde. Vértices, Campos dos Goytacazes/RJ, v. 12, n. 3, p. 17-40, 2010. 